\title{
A Textile-Based Wearable System for Vital Sign Monitoring: Applicability in Cardiac Patients
}

\author{
M Di Rienzo ${ }^{1}$, F Rizzo $^{1}$, G Parati $^{2}$, M Ferratini $^{3}$, \\ $\mathrm{G}_{\text {Brambilla }}{ }^{3}$, P Castiglioni ${ }^{1}$ \\ ${ }^{1}$ Centro Di Bioingegneria, Fnd Don C. Gnocchi, Milano, Italy \\ ${ }^{2}$ Universita' Milano Bicocca and Istituto Auxologico Italiano, Milano, Italy \\ ${ }^{3}$ Cardiac Rehabilitation Unit, Fnd Don C. Gnocchi, Milano, Italy
}

\begin{abstract}
We presents a new textile-based wearable system, named MagIC (Maglietta Interattiva Computerizzata), for the unobtrusive recording of cardiorespiratory and motion signals during spontaneous behavior in daily life and in a clinical environment. MagIC is composed of a vest, including textile sensors for ECG and breathing frequency detection, and a portable electronic board for motion assessment, signal preprocessing and wireless data transmission to a remote computer. MagIC has been tested in freely moving subjects at work, at home, while driving and cycling. Applicability of the system in a clinical setting is now under evaluation. Recordings performed on cardiac inpatients in bed and during physical exercise showed 1) good signals quality; 2) a correct identification of arrhythmic events; and 3) a correct estimation of the tacogram. These results encourage the further tuning of the MagIC system for a routine use in the clinical practice.
\end{abstract}

\section{Introduction}

There is now an increasing demand for smart wearable devices capable to detect vital signs while the subject is at work, during sport activities, at home or in a clinical environment without interfering with his/her spontaneous behavior. Addressing this issue implies to cope with problems concerning the design of new sensors, handling of possible noisy data, reduction in size, power consumption and costs of the hardware. A significant step forward in this area dates back to 1996 when at the MIT, researchers foresaw the development of garments capable to provide an unobtrusive monitoring of biological signals through textile sensors [1]. Since then, several groups in the world [2, 3, 4], including our laboratory at the Centro di Bioingegneria of the Don Gnocchi Fnd in Milan, have been working to translate this innovative concept into a real device.

The prototype of our wearable system for the unobtrusive recording of cardiorespiratory and motion signals during spontaneous behavior, is now available and uder test.

In the following, the main features of the new device, named MagIC (Maglietta Interattiva Computerizzata), are presented along with the first results concerning its application in a clinical environment.

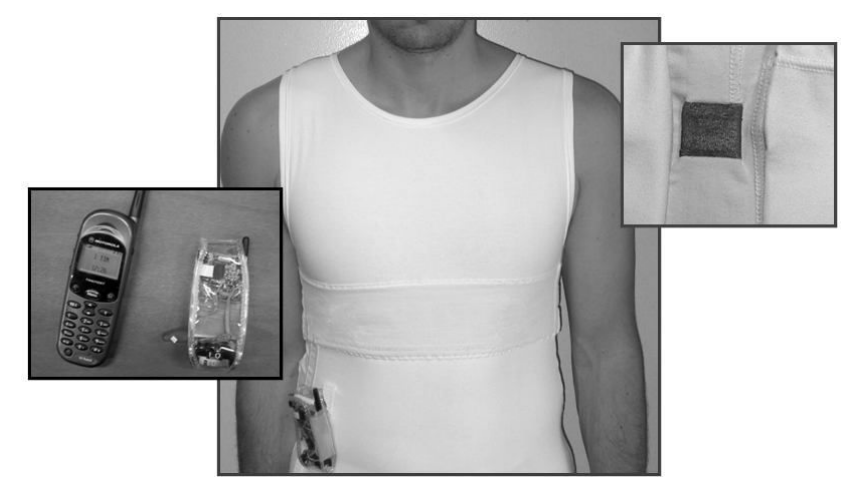

Figure 1. The MagIC system.

\section{Methods}

\subsection{System description}

The MagIC System is composed of a vest including textile sensors and a portable electronic board (see fig.1). The vest is mainly made of cotton and lycra and is fully washable. Through a CAD/CAM process two woven electrodes made by conductive fibers are located at the thorax level so to obtain an ECG lead. The contact between textile electrodes and the thorax is guaranteed by the elastic properties of the garment, without requiring application of gel or of any other medium. The vest also includes a textile-based transducer, obtained by a patented processing of the conductive fibers, for the assessment of respiratory frequency. Through connections still obtained by using the same conductive fibers, ECG and respiratory signals fed a portable electronic module - having the 
typical size and weight of a small cell phone - which is placed on the vest through a velcro strip. The electronic board detects also the subject's movement through a 2axis accelerometer and transmits all signals via a wireless connection to a remote computer or a PDA for data visualization and storage on disk.

\subsection{System validation}

The validation activity was aimed at evaluating the performances of the MagIC system in a clinical environment in terms of: 1) signal quality and artifact rate; 2) identification of rhythm abnormalities; 3) accuracy in estimating the beat-to-beat series of R-R intervals (RRI).

For the study, 9 healthy subjects and 14 cardiac inpatients of the Cardiac Rehabilitation Unit of Fondazione Don Gnocchi were enrolled.

Healthy subjects underwent two classic exercise tests: a 6-minute walk at constant speed and/or an incremental exercise tests at the cycloergometer. Signals from cardiac inpatients were recorded while the subjects were at rest in bed, and while performing a physical exercise on a cycloergometer as part of his/her rehabilitation program.

In all subjects ECG signals were simultaneously obtained from the MagIC System and a traditional ECG recorder (Cardioline Delta1 Plus, REMCO Italia, S. Pedrino di Vignate, Italy).

Evaluation of signal quality and artifact rate was obtained in healthy subjects and inpatients by measuring the percentage of the recorded signal in which QRS complexes could be correctly identified in the ECG of the MagIC system.

The capability of the system to identify rhythm abnormalities, was validated by letting experts to visually inspect the ECGs simultaneously recorded in cardiac inpatients from the MagIC system and from the traditional device .

The accuracy of the MagIC system in the estimation of the beat-to-beat RRI time series was evaluated for each incremental exercise test by Bland and Altman plots [6] and by measuring the MSE in each subject

\section{Results}

Results from the signal quality validation are summarized in table I.

Table I

\begin{tabular}{l|l|c}
\hline Group & Activity & Good signal (\%) \\
\hline Healthy & Increm. Exercise & $100 \%$ \\
Subjects & 6-min walk & $99 \%$ \\
\hline Cardiac & Bedrest & $99 \%$ \\
Patients & Exercise & $97 \%$ \\
\hline
\end{tabular}

Data showed that MagIC provided readable signals for more than $99 \%$ and $97 \%$ of the time while the subjects where lying supine and on the cycloergometer respectively. The study also showed the capability of the system to correctly identify the occurrence of atrial fibrillation and atrial or ventricular ectopic beats. Figure 2 shows a ventricular event as detected by the MagIC system and by the traditional ECG device.

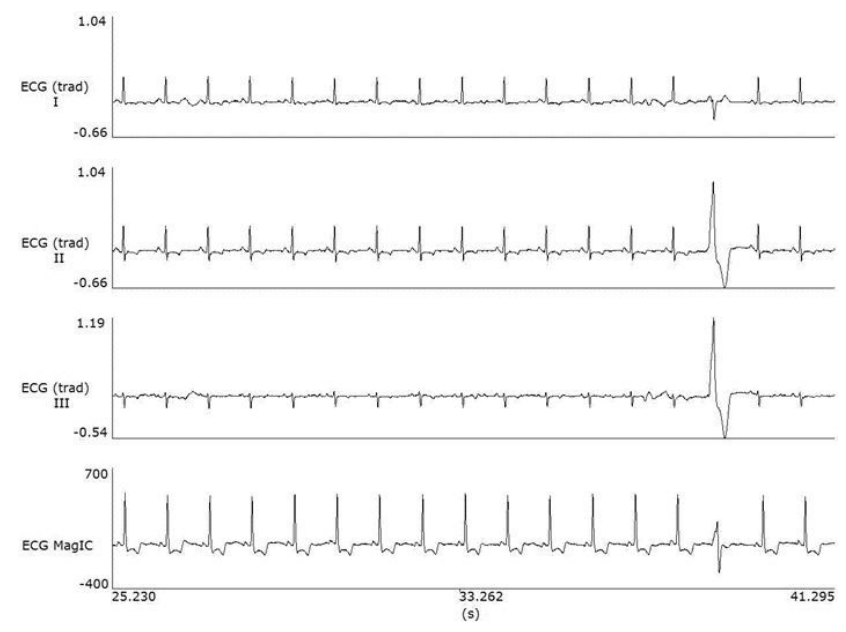

Figure 2. Example of a ventricular ectopic beat episode as recorded by a traditional 3-leads ECG chart (upper panels) and by the MagIC system (lower panel).

Fig.3 shows the tachograms simultaneously obtained from the MagIC System and a traditional ECG recorder in one subject during the incremental exercise test. The close similarity between the two profiles indicates a correct estimation of RR interval from the MagIC System also during exercise.

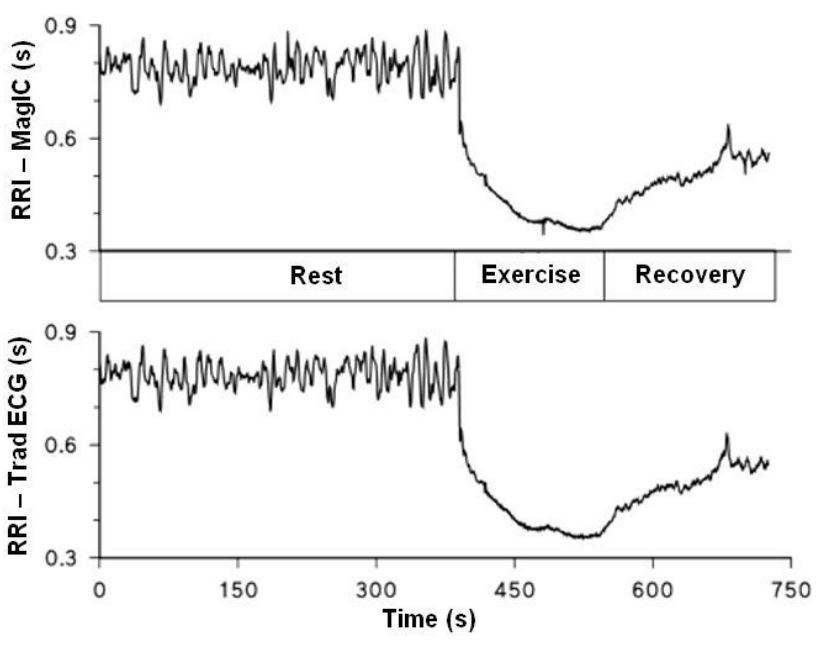

Figure 3. Tacograms from MagIC and traditional ECG during incremental exercise test 
This was confirmed by the Bland and Altman plots (see an example in fig.4), which in all subjects showed very small differences in the estimates obtained by the two systems (95\% limits of agreement within $1 \mathrm{~ms}$ ). The root mean squared error over the group was equal to 1.07 ms.

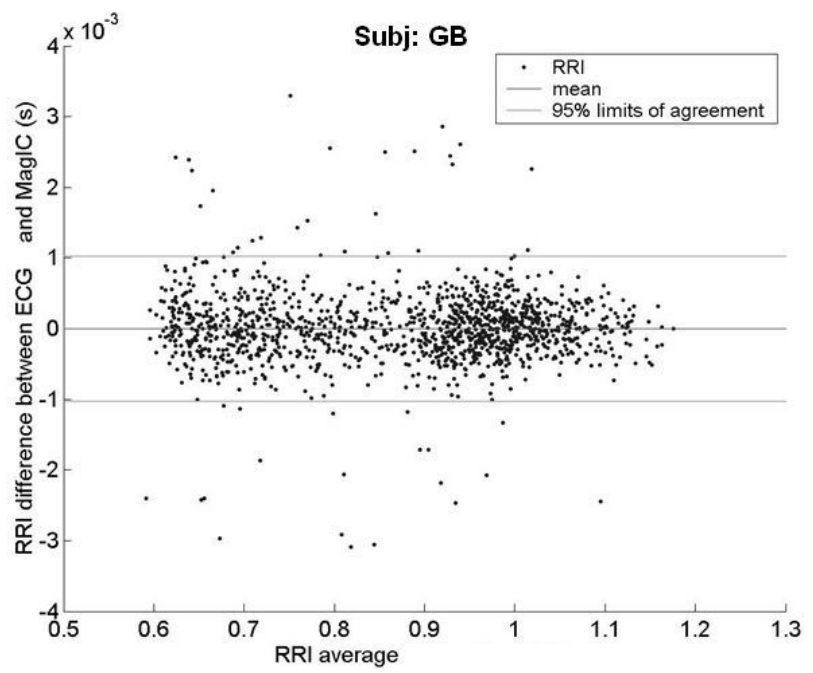

Figure 4. Bland and Altman plot (data collected during incremental exercise test)

\section{Discussion and conclusions}

A new wearable device for the assessment of vital signs based on textile sensors has been developed and is currently under test. The first positive results obtained from the clinical application of the MagIC System on cardiac patients do encourage the further development and tuning of the system for a routine use in the clinical setting and in daily life conditions. From a methodological point of view, our results also indicate that textile technology may provide an effective contribution in designing innovative devices for the assessment of biological signals.

\section{References}

[1] Post ER, Orth M, 'Smart Fabric, or "Wearable Clothing", Proc. of International Symposium on Wearable Computers. October 13 - 14, 1997. Cambridge, Massachusetts, p. 167$168 \mathrm{G}$.

[2] SmartShirt ${ }^{\mathrm{TM}}$, Sensatex, USA. Available: www.sensatex.com.

[3] LifeShirt, VivoMetrics, USA. Available: www.vivometrics.com

[4] Della Santa A, Mazzoldi A, De Rossi D. Dressware: wearable hardware. Mater Sci Eng 1999; C7:31-37.

[5] Bland JM, Altman DG. Measuring agreement in method comparison studies. Stat Methods Med Res 1999; 8(2):135160

Address for correspondence

Dr. Marco Di Rienzo

Centro di Bioingegneria, Fondazione Don C. Gnocchi

via Capecelatro 66

20133 Milano (Italy)

mdirienzo@cbi.dongnocchi.it 